\title{
CORRESPONDENCE Bacterial-viral filters to limit the spread of aerosolized respiratory pathogens during neonatal respiratory support in a pandemic era
}

Pediatric Research (2021) 89:1322-1325; https://doi.org/10.1038/s41390020-1102-4

\section{SCOPE OF THE DOCUMENT}

The novel coronavirus disease 2019 has raised several questions about how to limit the spread of highly transmissible respiratory viruses. While guidelines and recommendations exist for adult patients, these cannot be extrapolated to infants and neonates, as neonatal respiratory support presents specific issues (e.g., high apparatus dead space compared to neonatal airways, high bias flows, humidified gases, etc.) that make the transmission of respiratory pathogen control particularly challenging. Three recent papers address the issues of pathogen transmission, aerosol-generating procedures, and infection control before, during, and after neonatal resuscitation ${ }^{1,2}$ and during different respiratory support modes in the neonatal intensive care unit. ${ }^{3}$ This document provides more detailed indications on how to use bacterial-viral filters to reduce the spread of respiratory pathogens from neonatal patients with acute respiratory infections receiving respiratory support, with a focus on the protection of the environment and healthcare workers.

\section{INTRODUCTION TO AIR FILTERS}

Bacterial-viral air filters are medical devices used in respiratory ventilators or breathing circuits to protect patients, equipment, and/or the environment from viruses and bacteria. They may be either electrostatic or mechanical, based on their working principle: electrostatic filters use an induced electrostatic charge to capture particles, while mechanical filters use a pleated porous membrane. Mechanical filters can reach higher filtration efficiency than electrostatic filters, but they impose higher airflow resistance. Air filters are classified upon their efficiency: efficiency particulate air (EPA), high-efficiency particulate air (HEPA), and ultra-low penetration air filters retain a minimum of $99.95 \%, 99.97 \%$, and $99.999 \%$ of $0.3 \mu \mathrm{m}$ particles, respectively. Heat moisture and exchangers (HMEs) retain heat and humidity from exhaled air and return them to the patient during the following inspiration. HMEs also provide a filtration function that can be either electrostatic or mechanical, and they can be classified as either EPA or HEPA based on their filtration efficiency. HMEs are passive humidifiers and, as such, they should be placed at the inlet of the airway interface. HMEs must not be used with humidified gasses because the humidity retained by the hygroscopic membrane may increase airflow resistance. ${ }^{4}$

Connecting a bacterial-viral filter to the breathing circuit modifies its mechanical characteristics: (1) it increases the compliance of the circuit; (2) it increases dead space if placed at the airway interface; (3) it adds a resistance that causes a pressure drop between the inlet $\left(P_{1}\right)$ and the outlet $\left(P_{2}\right)$ of the filter:

$P_{2}=P_{1}-R \times V^{\prime}$.

The pressure drop increases with increasing filter resistance $(R)$ and with flow rate $\left(V^{\prime}\right)$. The filter resistance may increase as particles and humidity are retained.

\section{FILTERS WITHIN DOUBLE-LIMB BREATHING CIRCUITS}

Air filters may be connected in different positions within a doublelimb breathing circuit (Fig. 1).

\section{Position 1}

The bacterial-viral filter on the inspiratory limb has two functions: (1) protecting the equipment from the rare event of contamination with exhaled air, (2) protecting the patient in case he/she breathes room air through the safety valve that some ventilators open in case of sudden failure. The inspiratory filter does not prevent environmental contamination. The mechanical characteristics of inspiratory filters remain stable over time because the gas flowing through them is clean and dry. The pressure drop across the filter may affect the inspiratory flow and pressure waveforms. The high bias flows typically used in neonatal ventilators cause an additional pressure drop. Nevertheless, the alteration of inspiratory waveforms and the pressure drop associated with the bias flow should not significantly affect ventilation. If the ventilator measures the airway pressure at the Y-piece, it automatically compensates for the filter load. If not, the breathing circuit should be calibrated with the filter in place, so that the ventilator estimates the mechanical properties of the breathing circuit and compensate measurements accordingly.

\section{Position 2}

A filter between the breathing circuit and the airway interface protects the patient, equipment, and environment from airborne contamination. ${ }^{5,6}$ HMEs should be placed only in position 2 . This configuration increases dead space, thus affecting gas exchange. In adults, a filter between the Y-piece and the airway interface increases minute ventilation or arterial partial pressure of carbon dioxide. ${ }^{7}$ Position 2 should be avoided in neonates because filter dead space (e.g., $8-10 \mathrm{~mL}$ for the smallest filters) is very high compared with the patient tidal volume. ${ }^{8}$ The ventilator cannot detect the effects of a filter between the Y-piece and the airway interface. Therefore, monitoring ventilation waveforms over time is recommended to identify the possible consequences of increased filter resistance.

Positions 3 and 4

Expiratory filters prevent bacteria or virus transmission to the environment. They do not add dead space, but they increase 


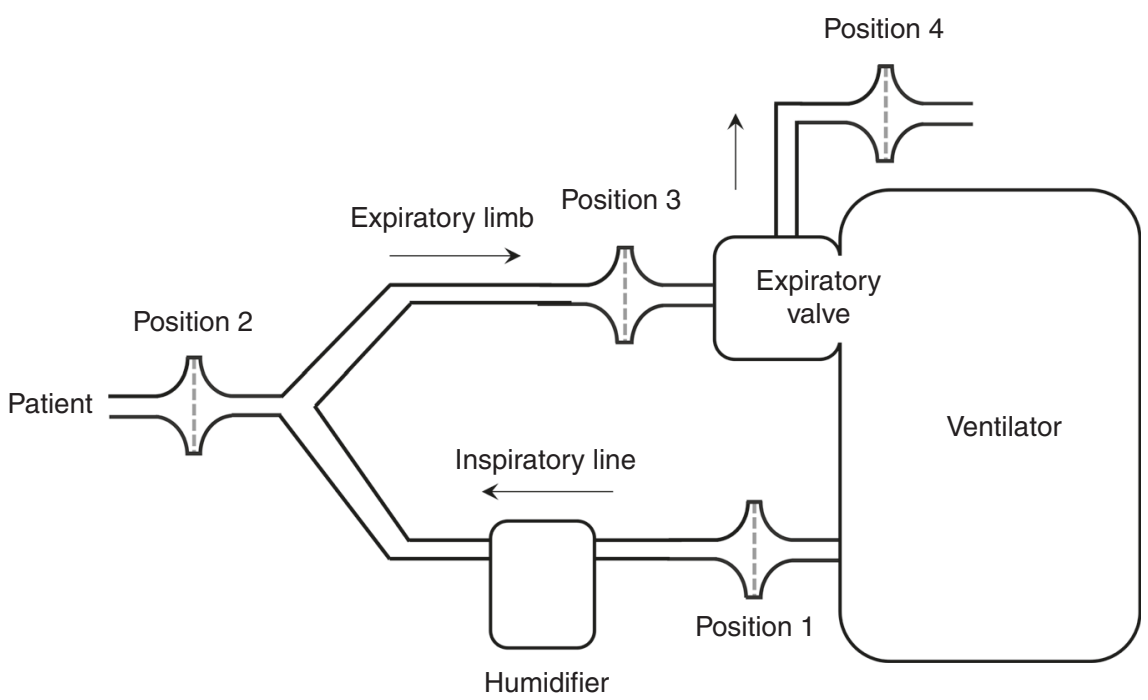

Fig. 1 Possible positions of the bacterial-viral filter within a double-limb breathing circuit.

circuit compliance and resistance, similarly to inspiratory filters. While the work required to overcome the resistance of the inspiratory line is provided by the ventilator, increasing the expiratory line resistance prolongs the time to exhale. This issue is particularly critical in patients at risk of developing intrinsic positive end-expiratory pressure (PEEP; e.g., patients with a small endotracheal tube or with obstructive diseases). The humidity of the gas flowing through the expiratory filter may significantly increase its resistance and nearly occlude it. ${ }^{4}$ The highest risk occurs using an HME filter in position 3 or 4 with humidified gasses and non-heated expiratory lines. ${ }^{9-11}$ HME filters must not be used with humidified gasses and must be connected only in position 2. If the expiratory limb of the breathing circuit is not heated, a water trap on the expiratory line may reduce the risk of rainout inside the filter. While the filter is in place, check that the airway pressure reaches the pre-set PEEP level and set the "high PEEP" alarm, if present, at a value only slightly higher than the desired PEEP. The expiratory filter is a single-patient device; it should also be changed if PEEP tends to increase compared with the pre-set value, in the presence of rainout, after nebulization, and, in any case, every $12-24 \mathrm{~h}$. Caution should be used during filter replacement because opening the breathing circuit is an aerosol-generating procedure. The resistance of the filter connected in position 3 produces a pressure drop between the patient's airways and the inlet of the expiratory valve, which controls the pressure inside the breathing circuit. Therefore, we recommend the use of the expiratory filter in position 3 if the ventilator measures airway pressure at the Y-piece. If the ventilator does not have a proximal pressure port, connecting the filter at the outlet of the expiratory valve (position 4) allows a more accurate estimate of the pressure applied to the patient. Connecting the filter in position 4 is possible only if the outlet of the expiratory valve is channeled to a duct.

Always refer to the user's manual before connecting the bacterial-viral filter to the ventilator or contact the manufacturer. Different indications for different ventilators are reported. The user's manuals refer to an inspiratory filter for Babylog 8000+ and VN500 (Dräger Medical AG \& Co, Lübeck, Germany), Servo-N (rev. 1.14) (Maquet, Solna, Sweden), Leoni+ (rev. 2.5.3) (Heinen+Löwenstein, Bad Ems, Germany), SLE 4000/5000 and 6000 (rev. UM131 Issue 4 for SLE 4000/5000 and UM154 Issue 6 for SLE 6000) (SLE UK, Croyden, United Kingdom), and Hamilton G5, C1, and MR1 (Hamilton Medical AG, Rhäzüns, Switzerland). The user's manual does not mention inspiratory filters for Fabian (rev. 113003.IT) (Acutronic, Hirzel, Switzerland), Avea (rev. J) (CareFusion, San Diego, CA), Giulia (rev. 2.3) (GINEVRI srl, Albano Laziale, Rome, Italy), and Stephanie and Sophie ${ }^{12}$ (Fritz Stephan GmbH, Gackenbach, Germany). The use of an expiratory filter is suggested for Avea, Servo-N, Leoni+, SLE ventilators, Giulia, Hamilton ventilators, Stephanie, and Sophie but not for Babylog 8000, VN500, and Fabian.

Leoni+, SLE ventilators, Fabian, Hamilton ventilators, and Giulia measure airway pressure with a proximal pressure line, while Babylog 8000+, VN500, Avea, Servo-N, Stephanie, and Sophie do not.

\section{BACTERIAL-VIRAL FILTERS DURING MANUAL VENTILATION}

Self-inflating bag

A filter between the self-inflating bag and the airway interface represents the easiest solution to protect patients, equipment, and the environment from contamination. Use a filter with the lowest possible dead space. Neonatal HME filters may have a dead space as low as $8-10 \mathrm{~mL}$. We suggest using an HME filter in this configuration because gasses are not humidified.

\section{T-piece}

The high bias flow flowing through the PEEP valve of the T-piece may produce aerosolized pathogens that are dispersed at a long distance toward the operator. In a standard T-piece, the only possible filter position is at the inlet of the airway interface. Since this configuration adds dead space, we propose an alternative solution, which consists in using a double-limb circuit, connecting the T-piece PEEP valve at the end of the expiratory line, and closing the patient outlet of the T-piece (Fig. 2).

\section{NON-INVASIVE VENTILATION}

Non-invasive respiratory support is an aerosol-generating procedure, and caution is required when managing it. We suggest administering non-invasive respiratory support using a mechanical ventilator with a double-limb breathing circuit and an expiratory filter connected as described above, whenever possible. To our knowledge, no studies evaluated the dispersion of aerosolized pathogens while using jet systems (e.g., Benveniste valve or Infant Flow Driver system) or bubble continuous positive airway pressure. These devices may increase pathogen dispersion, and 


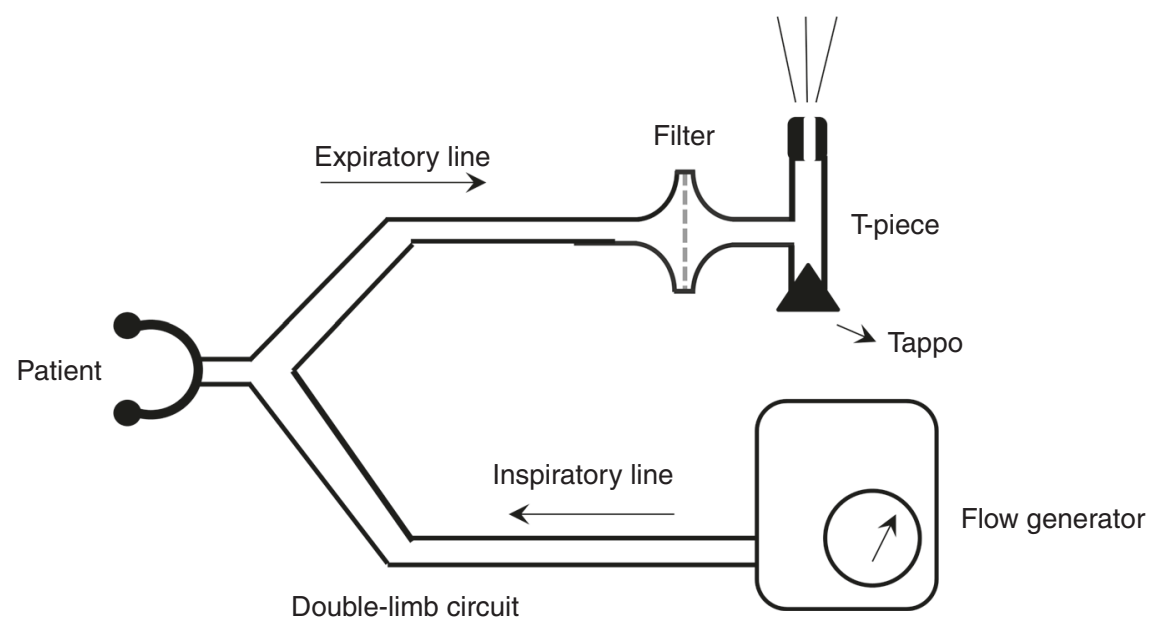

Fig. 2 Possible connection of an expiratory filter to a T-piece respirator.

connecting an expiratory filter to them is not feasible. In adults, high flow nasal therapy does not increase the risk infection, ${ }^{13-15}$ if associated with good seal. ${ }^{13}$ However, in neonates, a gas leak from the nares should be allowed. During non-invasive respiratory support, pathogens may spread through nose and mouth leaks, thus covering the infant's nose and mouth with a surgical mask may trap or reduce the velocity of airborne particles. Non-invasive respiratory support should be administered in appropriate airborne isolation rooms.

\section{FUTURE PERSPECTIVES}

Future studies should evaluate the extent of pathogen dispersion during different types of neonatal respiratory support and should investigate whether the use of viral/bacterial filters in the breathing circuit can significantly affect ventilation and reduce the spread.

\section{ACKNOWLEDGEMENTS}

We are grateful to Maurizio Ginepro, Ettore Legranzini, Federico Araldi, and Giovanni Poldi for their contribution. No financial assistance was received in support of the study.

\section{AUTHOR CONTRIBUTIONS}

E.Z. gave substantial contributions to the conception and design of the manuscript and acquisition of literature data; she drafted the article and gave her approval of the manuscript version to be published. C.V. gave substantial contributions to the conception and design of the manuscript and acquisition of literature data; she helped in drafting the article and gave her approval of the version to be published. R.D. gave substantial and original contributions to the conception and design of the manuscript; he revised the manuscript critically for important intellectual content and gave his approval of the version to be published. F.M. revised the manuscript critically for important intellectual content and gave his approval of the version to be published. C.G. gave substantial contributions to the conception and design of the manuscript and in revising the manuscript critically for important intellectual content; she helped in drafting the article and gave her approval of the version to be published. M.L.V. gave substantial and original contributions to conception and design of the manuscript and acquisition of literature data; she revised the manuscript critically for important intellectual content and gave her approval of the version to be published.

\section{ADDITIONAL INFORMATION}

Competing interests: The authors declare no competing interests.

Patient consent: The authors declare that patient consent was not required.
Publisher's note Springer Nature remains neutral with regard to jurisdictional claims in published maps and institutional affiliations.

Emanuela Zannin ${ }^{1}$, Chiara Veneroni ${ }^{1}$, Raffaele L. Dellaca ${ }^{1}$, Fabio Mosca ${ }^{2}$, Camilla Gizzi ${ }^{3}$ and Maria Luisa Ventura ${ }^{4}$

${ }^{1}$ TechRes Lab, Dipartimento di Elettronica, Informazione e Bioingegneria, Politecnico di Milano, Milan, Italy; ${ }^{2}$ Neonatal Intensive Care Unit, Fondazione IRCCS Ca' Granda-Ospedale Maggiore Policlinico, Milan, Italy; ${ }^{3}$ Neonatal Intensive Care Unit, "San Giovanni Calibita Hospital" Fatebenefratelli - Isola Tiberina, Rome, Italy and ${ }^{4}$ Neonatal Intensive Care Unit, Fondazione MBBM-ASST-Monza, Monza, Italy Correspondence: Camilla Gizzi (camillagizzi@gmail.com)

\section{REFERENCES}

1. Trevisanuto, D. et al. Neonatal resuscitation where the mother has a suspected or confirmed novel coronavirus (SARS-CoV-2) infection: suggestion for a pragmatic action plan. Neonatology 24, 1-8 (2020).

2. Chandrasekharan, $\mathrm{P}$. et al. Neonatal resuscitation and postresuscitation care of infants born to mothers with suspected or confirmed SARS-CoV-2 infection. Am. J. Perinatol. https://doi.org/10.1055/s-0040-1709688 (2020).

3. Shalish, W., Lakshminrusimha, S., Manzoni, P., Keszler, M. \& Sant'Anna, G. M. COVID19 and neonatal respiratory care: current evidence and practical approach. Am. J. Perinatol. https://doi.org/10.1055/s-0040-1710522 (2020).

4. Lawes, E. G. Hidden hazards and dangers associated with the use of HME/filters in breathing circuits. Their effect on toxic metabolite production, pulse oximetry and airway resistance. Br. J. Anaesth. 91, 249-264 (2003).

5. Berry, A. J. \& Nolte, F. S. An alternative strategy for infection control of anesthesia breathing circuits: a laboratory assessment of the Pall HME filter. Anesth. Analg. 72, 651-655 (1991).

6. Gallagher, J., Strangeways, J. E. M. \& Allt-Graham, J. Contamination control in long-term ventilation: a clinical study using a heat- and moisture-exchanging filter. Anaesthesia 42, 476-481 (1987).

7. Campbell, R. S., Davis, K., Johannigman, J. A. \& Branson, R. D. The effects of passive humidifier dead space on respiratory variables in paralyzed and spontaneously breathing patients. Respir. Care 45, 306-312 (2000).

8. King, M. R. \& Feldman, J. M. Optimal management of apparatus dead space in the anesthetized infant. Paediatr. Anaesth. 27, 1185-1192 (2017).

9. McEwan, A. I., Dowell, L. \& Karis, J. H. Bilateral tension pneumothorax caused by a blocked bacterial filter in an anesthesia breathing circuit. Anesth. Analg. 72, 440-442 (1993).

10. Smith, C. E., Otworth, J. R. \& Kaluszyk, P. Bilateral tension pneumothorax due to a defective anesthesia breathing circuit filter. J. Clin. Anesth. 3, 229-234 (1991).

11. Tonnelier, A., Lellouche, F., Alexandre Bouchard, P. \& L'Her, E. Impact of humidification and nebulization during expiratory limb protection: an experimental bench study. Respir. Care 58, 1315-1322 (2013).

12. Hummler, H. D. \& Poets, C. F. in Manual of Neonatal Respiratory Care (eds Donn, S. M. \& Sinha, S. K.) 413-429 (Springer Nature, 2017). 
13. Hui, D. S. et al. Exhaled air dispersion during high-flow nasal cannula therapy versus CPAP via different masks. Eur. Respir. J. 53, 1802339 (2019).

14. Kotoda, M. et al. Assessment of the potential for pathogen dispersal during highflow nasal therapy. J. Hosp. Infect. 104, 534-537 (2020).
15. Leung, C. C. H. et al. Comparison of high-flow nasal cannula versus oxygen face mask for environmental bacterial contamination in critically ill pneumonia patients: a randomized controlled crossover trial. J. Hosp. Infect. 101, 84-87 (2019). 